\title{
A Neural Network Model for Driver's Lane-Changing Trajectory Prediction in Urban Traffic Flow
}

\author{
Chenxi Ding, ${ }^{1,2}$ Wuhong Wang, ${ }^{1}$ Xiao Wang, and Martin Baumann ${ }^{2}$ \\ ${ }^{1}$ Department of Transportation Engineering, Beijing Institute of Technology, Beijing 100081, China \\ ${ }^{2}$ Institut für Verkehrssystemtechnik, Deutsche Zentrum für Luft-und Raumfahrt, \\ Lilienthalplatz 7, 38108 Braunschweig, Germany
}

Correspondence should be addressed to Wuhong Wang; wangwuhong@bit.edu.cn

Received 13 September 2012; Accepted 10 November 2012

Academic Editor: Huimin Niu

Copyright (C) 2013 Chenxi Ding et al. This is an open access article distributed under the Creative Commons Attribution License, which permits unrestricted use, distribution, and reproduction in any medium, provided the original work is properly cited.

\begin{abstract}
The neural network may learn and incorporate the uncertainties to predict the driver's lane-changing behavior more accurately. In this paper, we will discuss in detail the effectiveness of Back-Propagation (BP) neural network for prediction of lane-changing trajectory based on the past vehicle data and compare the results between BP neural network model and Elman Network model in terms of the training time and accuracy. Driving simulator data and NGSIM data were processed by a smooth method and then used to validate the availability of the model. The test results indicate that BP neural network might be an accurate prediction of driver's lane-changing behavior in urban traffic flow. The objective of this paper is to show the usefulness of BP neural network in prediction of lane-changing process and confirm that the vehicle trajectory is influenced previously by the collected data.
\end{abstract}

\section{Introduction}

The most crucial road traffic problems requiring solutions have been the reduction of traffic accidents and traffic congestion [1]. Increasing the safety level of driving in traffic, especially the safety of maneuvers such as lane-changing and overtaking, is one of the key technologies for the Intelligent Transportation System (ITS) to achieve congestion-free and accident-free traffic situations [2]. Every year, traffic accidents result in approximately 1.2 million fatalities worldwide; without new prevention measures, this number could increase by $65 \%$ over the next two decades [3]. Researchers estimate that lane-changing crashes account for $4 \%$ to $10 \%$ of all vehicle crashes in the USA [4]. Although, this value is not very high, the delay time it causes accounts for $10 \%$ of the total time caused by all traffic accidents.

Due to the importance of driving behavior to vehicle safety, many researchers have attempted to model driving behavior. A general trend in the study of modeling driving behavior is the greater application of computational artificial intelligence. Because the driver's mental and physical behavior is nondeterministic and highly nonlinear, it is difficult for traditional methods to embody this kind of uncertain relationship. The Artificial Neural Networks, fuzzy logic theory, and dynamic Bayesian networks, which include well-known hidden Markov models, have attracted many researchers to do relative research. Kumagai et al. focused on the prediction of drivers' intensions of stopping the car at an intersection with their current and historical maneuvers based on a simple dynamic Bayesian Network [5]. Tezuka et al. developed a method to infer driver behavior with a driving simulator to evaluate continuous time-series steering angle data at the time of lane-changing. The proposed method used a static type conditional Gaussian model on Bayesian Networks [6]. Kuge et al. proposed hidden Markov models (HMMs) using observations of vehicle parameters and lane positions to model trajectories [7]. Sathyanarayana et al. proposed a method to model driver behavior signals using hidden Markov models. The hierarchical framework and initial results can encourage more investigations into driver behavior signal analysis and related safety systems employing a partitioned submodule strategy [8]. Pentland and Andrew proposed that many human behaviors can be accurately described as a set of dynamic models sequenced 
together by a Markov chain. They considered the human as a device with a large number of internal mental states and used the dynamic Markov models to recognize human behaviors from sensory data and to predict human behaviors a few seconds into the future [9]. Macadam and Johnson demonstrated the use of elementary neural networks (a two-layer back propagation) to represent the driver's steering behavior in double lane-changing maneuvers and S-curve maneuvers. Due to the limited data source for neural networks, it was concluded that the adaptive nature of neural networks should be used for modeling driver steering behavior under a variety of operation scenarios [10]. Cheng et al. used a BackPropagation neural network as a controller for an automated vehicle system. Camera images were used as inputs to the neural network [11]. Tomar et al. proposed a method to give the future lane-changing trajectories accurately for discrete patches using a multilayer perceptron (MLP) [12]. The proposed multilayer perception network is a simple single input, single output based on a single hidden layer and is used for training, testing, and prediction of the vehicle trajectories.

Most of the prior research based on Neural Network or DBN (Dynamic Bayesian Network) have recognized temporal information, inferred current states, or simply detected the action after it has begun, but it has not predicted future states. Most studies until now have analyzed driver behavior by offline processing of data due to the limited datasets. Besides, some studies are only able to train itself and predict the future positions of a lane-changing vehicle in certain discrete sections of the path only and not over the complete lane change path. Therefore, our research is intended to realtime predict lane-changing trajectory based on a time delay Back-Propagation Neural Network (BPNN). We proposed two-layer Tansig and Linear BP neural network to deal with $n$-input, single-output problems. The position, velocity, acceleration, and time headway of the vehicle were used as the inputs of the model. The future lane-changing trajectory was considered as the desired output of the trained network. We mixed the data of the different path sections as inputs to train the network in order to make its applications more wide. To validate the model, we employed NGSIM trajectory data and a smoothing method. Simulation results demonstrate the effectiveness of the proposed model.

In this research, we attempted to answer the following questions based on the proposed model. Is it possible to infer future driving states in different path sections using one neural network? How long of the prediction time is stable? Is it necessary to combine various input data for the prediction, and which one (or combination) is the more informative indicator? How to post-processed the raw data effectively? Which network is suitable for online analysis, BP Neural Network or Elman Network?

\section{Traditional Lane-Changing Model Development}

A major problem in developing a driver model lies on the requirement of suitable quantitative formulations by means of mathematical and statistical theories [13-15].

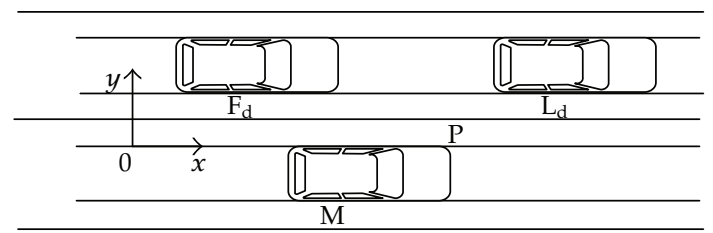

FIGURE 1: Prelane-changing configuration showing position of subject vehicle $M$.

Lane-changing behavior has been widely studied from the viewpoint of traffic flow theory. A typical lane-changing algorithm which is used for microscopic traffic simulation is described in this section [16].

Figure 1 illustrates the lane-changing situation for vehicle $M$. The vehicles $L_{d}, F_{d}$, and $M$ represent the leading vehicle in the destination lane, the following vehicle in the destination lane, and the subject vehicle, respectively. The trajectories of subject vehicle $\mathrm{M}$ can be constructed by a two-dimensional coordinate system.

The objective of this section is to use a simple lanechanging model to define the lateral acceleration, the lateral velocity, and the lateral distance of subject vehicle $M$ during a certain time-interval $\left[t_{0}, T\right]$ in Figure 2 . The time-intervals for the lane-changing maneuver are shown in Figure 2 and the times $t_{0}-T$ are defined as follows.

(i) At time $t_{0}$, the subject vehicle $M$ starts lane-changing maneuver and sets $t_{0}=0$.

(ii) At time $t_{\text {adj }}$, the subject vehicle $M$ adjusts successfully for lateral acceleration.

(iii) At time $t_{C}+t_{\text {adj }}$, the subject vehicle $M$ arrives at the marginal collision point.

(iv) At time $t_{\text {lat }}+t_{\mathrm{adj}}$, the subject vehicle $\mathrm{M}$ finishes the lateral acceleration.

(v) At time $T$, the subject vehicle $M$ finishes the lanechanging maneuver.

Note that with the exception of the subject vehicle, the lateral and longitudinal accelerations of the other vehicles are assumed to be 0 . Figure 3 illustrates the motion of vehicle $\mathrm{M}$ during a lane-changing or merge maneuver. $\theta(t)$ is the angle between the tangent of the lane changing trajectory at the time $t$ and the X-axis. $h$ is the lane width. $H$ is the total lateral displacement for vehicle M. Memar et al. developed a sinusoidal pattern of lateral acceleration of the subject vehicle [17]. Instantaneous lateral acceleration $a_{\text {lat }}(t)$ is given by (1). Where $H$ is the total lateral displacement for the subject vehicle $M, t$ is the elapsed time, and $t_{\text {lat }}$ and $t_{\text {adj }}$ are defined as above. According to (1), the lateral acceleration is positive within the first half of the lateral displacement, that is, $H / 2$, and negative in the second half.

On basis of the lateral acceleration, the lateral velocity and the lateral distance traveled of the front-left corner P during a lane-changing can be derived by successive integration, given in (2) and (3).

As indicated above, traditional lane-changing models do not consider the uncertainties and perceptions in human 


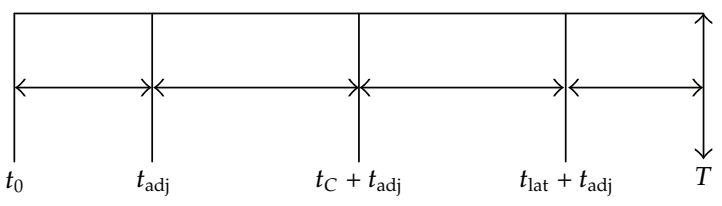

FIgURE 2: Time-intervals of lane-changing maneuver.

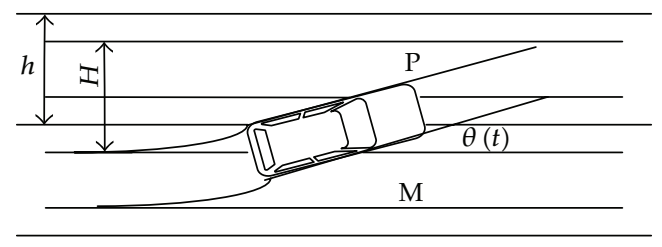

FIGURE 3: The vehicle M's motion during a lane-changing/merge maneuver.

behavior that are involved in modeling lane-changing. Therefore, research that develops lane-changing model from the artificial intelligence viewpoint is very important, such as the artificial neural network method introduced in the next section. The random term in network is attributable to a specific individual and the unimportant variables

$$
\begin{aligned}
& a_{\mathrm{lat}}(t)=\left\{\begin{array}{c}
2 \pi H / t_{\mathrm{lat}}^{2} \times \sin \left(\frac{2 \pi}{t_{\mathrm{lat}}}\left(t-t_{\mathrm{adj}}\right)\right), \\
t_{\mathrm{adj}} \leq t \leq t_{\mathrm{lat}}+t_{\mathrm{adj}}, \\
0, \quad \text { otherwise, }
\end{array}\right. \\
& v_{\text {lat }}(t)=\left\{\begin{array}{c}
-H / t_{\text {lat }} \times \cos \left(\frac{2 \pi}{t_{\text {lat }}}\left(t-t_{\text {adj }}\right)\right)+\frac{H}{t_{\text {lat }}}, \\
t_{\mathrm{adj}} \leq t \leq t_{\text {lat }}+t_{\text {adj }}, \\
0, \quad \text { otherwise, }
\end{array}\right. \\
& y_{\text {lat }}(t)=\left\{\begin{array}{l}
H, \quad t \geq t_{\text {lat }}+t_{\mathrm{adj}}, \\
-H / 2 \pi \times \sin \left(\frac{2 \pi}{t_{\text {lat }}}\left(t-t_{\mathrm{adj}}\right)\right)+\frac{H}{t_{\text {lat }}}\left(t-t_{\mathrm{adj}}\right), \\
t_{\mathrm{adj}} \leq t \leq t_{\mathrm{lat}}+t_{\mathrm{adj}},
\end{array}\right.
\end{aligned}
$$

\section{Neural Network Model for Lane-Changing Trajectory Prediction}

It is necessary that Advanced Driver Assistance System (ADAS) is provided with concepts and techniques that enable prediction of future situations. In order to optimize warning and control strategy of driver assistance systems, determine assistance systems more exactly, an analysis of driving behavior prediction under real-time traffic conditions is very essential to carry out driving erroneous analysis at the microscopic level. As an example, early recognition of a lane-changing behavior would help to adapt the warning and control strategy of Forward Collision Avoidance Assistance Systems and Lane Departure Warning (LDW) Systems.

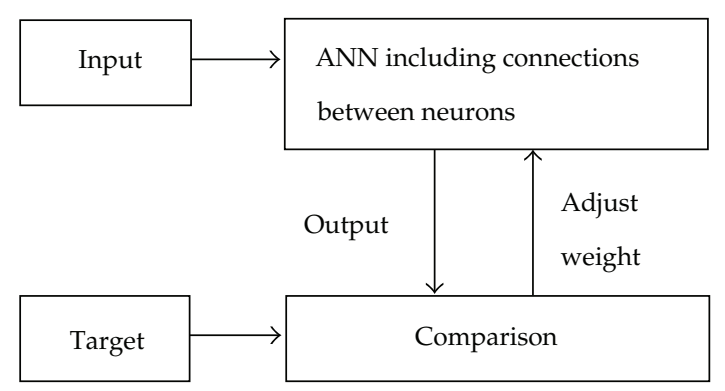

FIGURE 4: Conceptual operation of ANN model.

Therefore, our research objective was the development of a neural network-based model for collision avoidance systems in the case of lateral lane-changing and longitudinal car following. Consistent with this primary goal, different advanced neural network models were employed and compared for the same problem.

3.1. Artificial Neural Network. Artificial Neural Networks (ANNs) are massive parallel adaptive networks of simple nonlinear computing elements. These elements are called neurons and are intended to model some functionalities of the human nervous system in order to take advantage of its computational strength [18]. As a commonly used nonlinear function approximation tool, artificial neural network has shown great advantages in forecasting, pattern identification, optimization techniques, and signal processing for its nonlinear, flexible, and valid self-organization properties. A variety of problem areas are modeled using ANN [19-21] and in many instances, ANN has provided superior results compared to the conventional modeling techniques.

The basic model of ANN consists of computational units, which are a highly simplified model of the structure of the biological neural network [22]. Conceptual operation of ANN is shown in Figure 4. ANN is regarded as a black box that takes a weighted sum of all inputs and computes an output value using a transformation or output function. The output value is propagated to many other units via connections between units.

In general, the output function is a linear function in which a unit becomes active only when its net input exceeds the threshold of the unit, or it is a sigmoid function which is a nondecreasing and differentiable function of the input. Computational units in an ANN model are hierarchically structured in layers and depend upon the layer in which a unit resides. The units are called input, hidden, or output units. There are many input units, and some are dependent on the others. The output units are dependent on all input units. A hidden unit is used to augment the input data in order to support any required function from input or output. The inputs and outputs can be discrete or continuous data values. The input and output could also be stochastic, deterministic, or fuzzy.

In order to store a pattern in a network, it is necessary to adjust the weights of the connections in the network. The set of all weights on all connections in a network form a weight vector. The process of computing appropriate weights is called 
a learning law or learning algorithm. The learning process of ANN can be thought of as a reward and punishment mechanism [23], whereby when the system reacts appropriately to an input, the related weights are strengthened. In this case, it is possible to generate outputs, which are similar to those corresponding to the previously encountered inputs. On the contrary, when undesirable outputs are produced, the related weights are reduced. The model learns to give a different reaction when similar inputs occur, thus updating the system towards producing desirable results, whilst the undesirable ones are "punished."

Back-Propagation (BP) neural network, a typical case of neural networks, is used most widely and is more mature than other networks. BP neural network models consist of an input layer, one or several hidden layers, and an output layer. The typical BP neuron model is shown in Figure 5. When a set of input values and corresponding desired output values are supplied to the network, the transferred value is propagated from the input layer through hidden layers to the output layer. The neural network tries to learn the input-output parameter relationship process by adapting its free parameters.

Mathematical expression of a BP neural network is defined as

$$
Y_{j}=f\left(\sum_{i=1}^{n} w_{j i} x_{i}-b_{j}\right)=f\left(n_{j}\right)
$$

where column vector $\mathbf{X}$ is input vector, row vector $\mathbf{W}_{j}$ is the connection weight vector for neuron $j, b_{j}$ is the threshold of the output, $n_{j}$ represents the input of neurons, and the function $f$ is the transfer function.

\subsection{BP Neural Network Model for Lane-Changing Trajectory}

Prediction. For safe driving, it is necessary that the drivers perceive the relevant objects of a situation, comprehend the meaning of these objects to form a holistic understanding of the current situation, and predict the future development of the situation [24]. However, it is difficult for traditional lane-changing behavior model to embody the uncertainty in the series of cognitive behavior of drivers. Unlike the classic mathematic methods, BP networks can approximate the specific inputs and outputs relationship without a certain model.

Our research has attempted to set up a BP neural network model for lane-changing trajectory prediction and approximate the simulation of vehicle-to-vehicle interactions during the lane-changing process. Figure 6 shows a simple lanechanging maneuver.

In this study, an $n$-input, single-output based time delay BP neural network with two hidden layers is used for training, testing, and prediction of the vehicle trajectories. There are 40 sets of training samples consisted of four variables: the prior position, velocity, acceleration of the object vehicle, and the time headway. Each input variable consists of 1 second contiguous time history sets (or 10 frames). The desired output is the prediction of the next state 1 second in the lanechanging process by delaying time. The network was trained based on the Levenberg-Marquardt algorithm and the meansquared error was examined. Network weight values are then

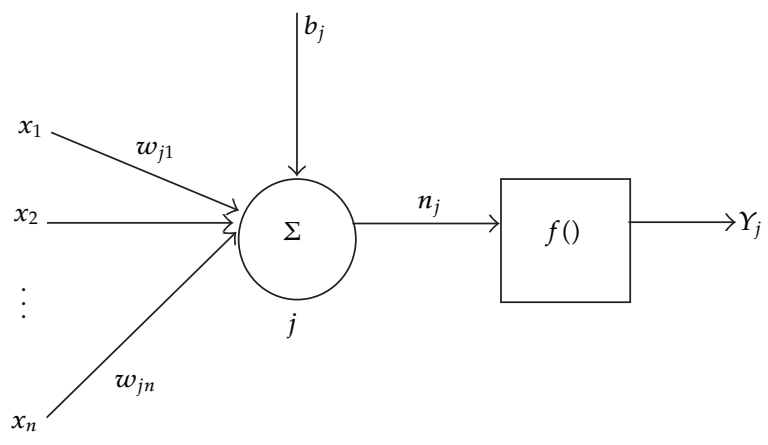

FIgure 5: Typical BP neuron model.

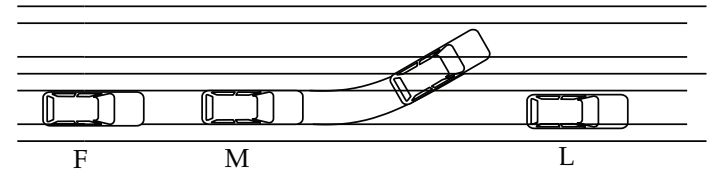

FIgure 6: The vehicles M, F, and L during a lane-changing maneuver.

iteratively adjusted until output errors are minimized. Once appropriate training data were collected, learning procedure could be implemented in order to reach similar performance with neural networks. Note that we mixed the training data of the different path sections as inputs to make the applications more wide.

\section{Simulation Results and Discussion}

4.1. Date Collection Based on Driving Simulation Test. In the simulation test, a driving simulator was used to collect driving behavior data including vehicle movements and maneuver operations. Separate computers were used to generate vehicle motion calculations and the front view displays. Subjects were instructed to use the driving simulator executing lanechanging and overtaking maneuvers on a two-lane road. There were 32 male drivers and 8 female drivers, their ages range from 24 to 50 years old and driving experience range from 1 year to 23 years. All the training data for the BP neural network was obtained from a driving simulator.

4.2. Results and Discussion of BP Neural Network Model. The BP neural network model used to predict lane-changing trajectory is shown in Figure 7. The network model consists of an input layer, two hidden layers, and an output layer. A nonlinear sigmoid defines each neuron's activation function in the first layer. The second layer neurons are linear. The biases $b$ is attributable to a specific individual, uncertainties, and the unimportant variables.

In this paper, we only show tested results of lane-changing lateral trajectories as an example. Figure 8 shows the tested lateral trajectory from a driving simulator and the predicted lateral trajectory from BP neural network model. The prediction of the vehicle trajectory has large error in the initial phase because of the small number of previous samples. 


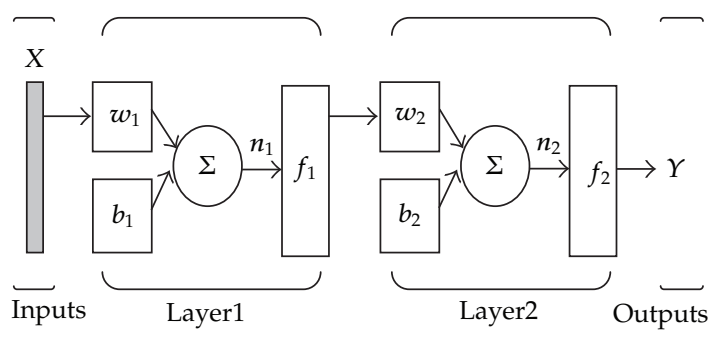

FIGURE 7: BP neural network with two hidden layers.

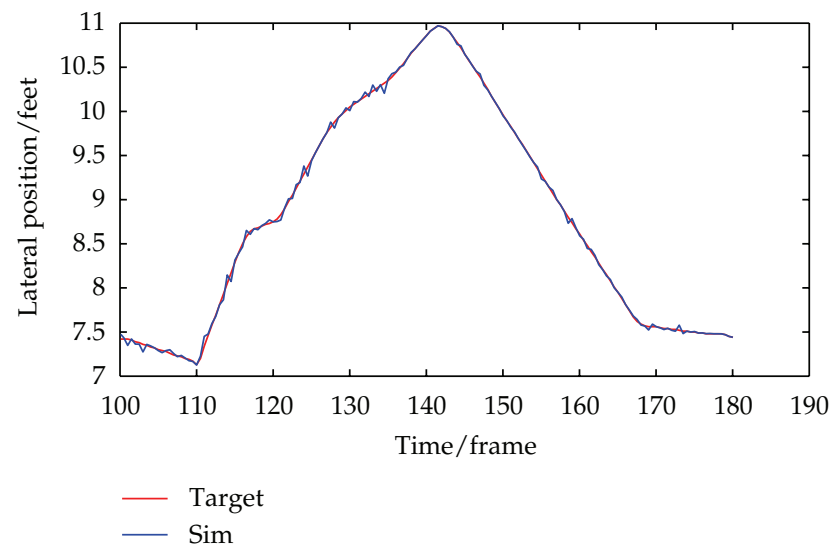

FIGURE 8: Predicted lateral trajectory of the vehicle based on BPNN with 25 hidden nodes.

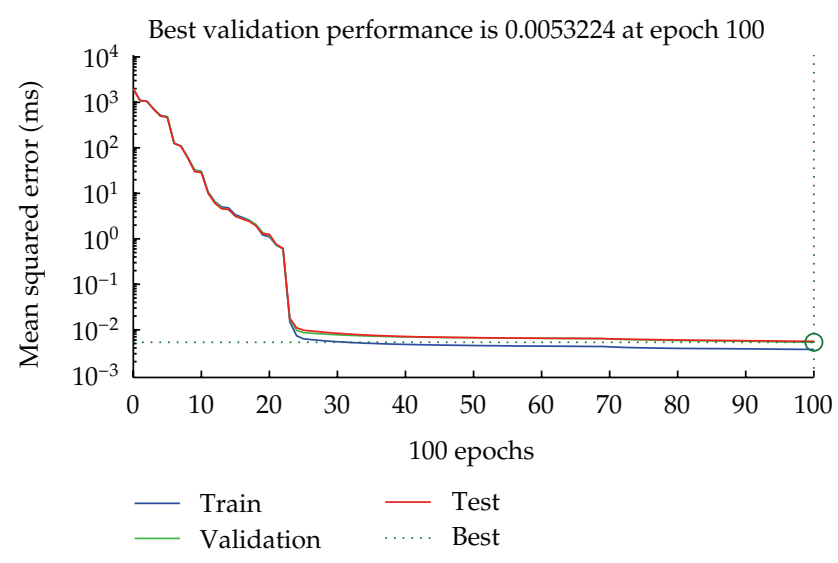

FIGURE 9: Performance curve of BPNN.

Performance curve of BPNN is shown in Figure 9. As indicated in Figure 9, by increasing the number of iterations, the performance of the network will be improved. But when the number of iterations is large enough, an increase in the number of iterations will no longer reduce the error rate. Besides, we also found vehicle lateral trajectory is influenced by time headway and the prior lateral trajectory more obviously. It is reasonable, because most of lane-changing maneuvers are caused by a slow leading vehicle.

4.3. Model Validation Based on NGSIM Data. The Next Generation Simulation (NGSIM) data is used to test and verify our model. NGSIM data provides detailed vehicle trajectory data, wide-area detector data, and supporting data needed

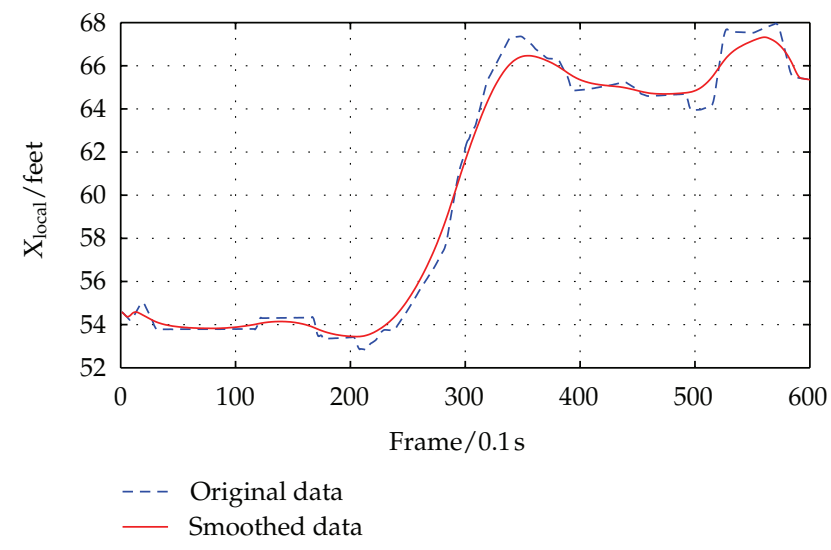

FIGURE 10: Smoothed lateral position.

for behavioral algorithm research. Considering observations in real traffic are always affected by measurement errors, we smooth the raw data which is used to test the model.

Taking the lateral position and vehicle velocity, for example, the smooth results are shown in Figures 10 and 11. The test results of different prediction time are shown in Figure 12. The green curve is lateral position with $2 \mathrm{~s}$ prediction time (MSE $=0.2273)$. The blue curve is lateral position with $1 \mathrm{~s}$ prediction time (MSE $=0.0184$ ), which is more accurate.

The validation results demonstrate the effectiveness of BNs model around a prediction time of $1 \mathrm{~s}$. And validations using a new sample of NGSIM also prove that our model can predict the future positions of a lane-changing vehicle under different path sections.

4.4. Comparison between BPNN and Elman Network. For the purpose of comparison, training time and accuracy are considered between the BPNN model and the Elman Network model. The training time is the time needed to train a neural network. The accuracy is measured by calculating the error for the testing data points. Elman Network differs from BPNN in that the first layer has a recurrent connection, shown in Figure 13. We employed the same transfer function, training algorithm, neural nodes, and training samples.

In Figures 14, 15, and 16, the comparison results with $1 \mathrm{~s}$ prediction time show that convergence rate is increased and the training time is reduced with the BPNN model. But the accuracy is increased with the Elman Network. The meansquared error based on BPNN model is 0.0371, whereas mean-squared error based on Elman Network model is 0.0279 .

Simulation results demonstrate that in this application BPNN is more advantageous to the training time and accuracy because of the simpler network structure. For the future research on real-time on-line prediction, both the training time and accuracy are important.

\section{Conclusion}

The main motivation for using a neural network is its ability to learn and incorporate the uncertainties from real driving data. This means, after learning from the driving behavior 


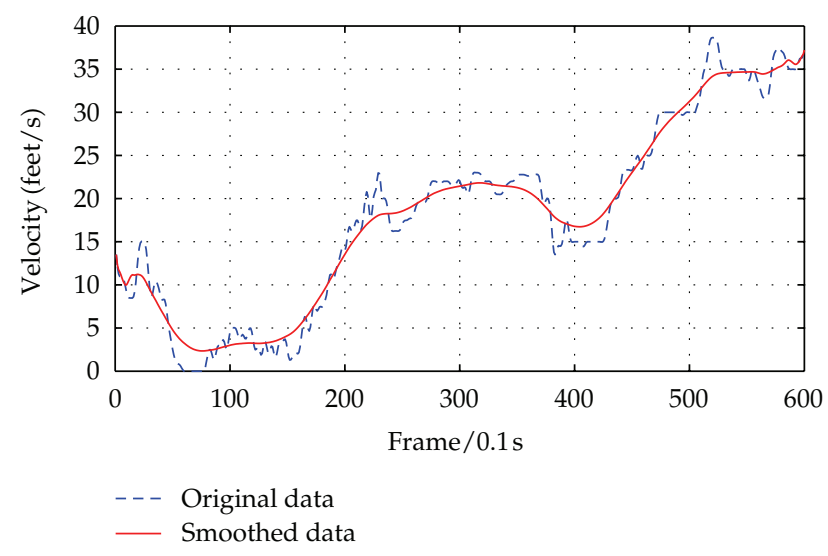

FIGURE 11: Smoothed vehicle velocity.

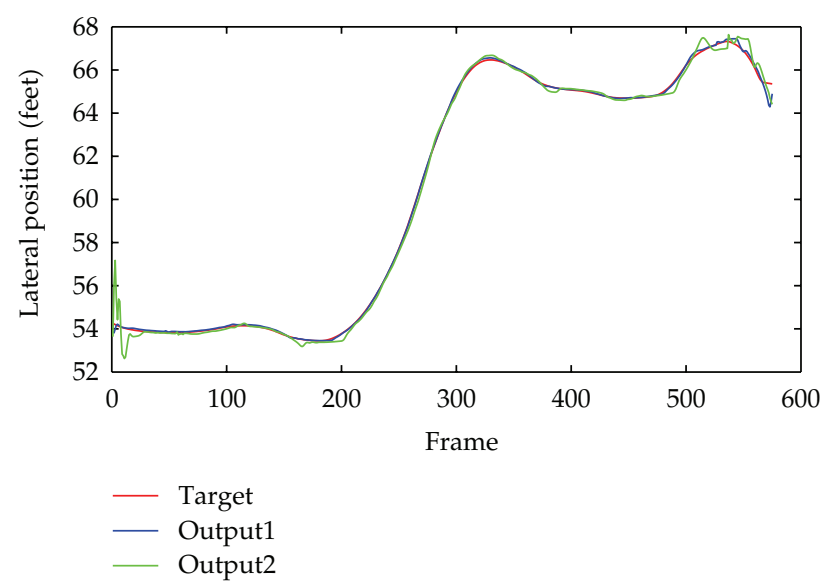

FIgURe 12: Predicted lateral trajectory of the vehicle with $1 \mathrm{~s}$ and $2 \mathrm{~s}$ prediction time.

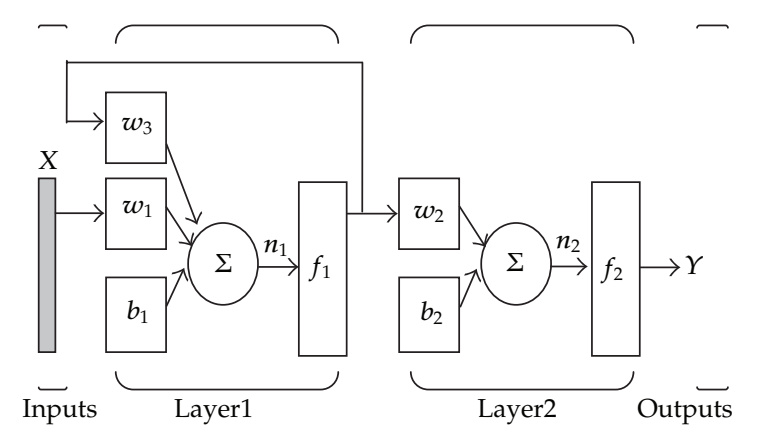

Figure 13: Elman Network with two hidden layers.

data, neural network could generate vehicles states to reproduce any style of driving. In this study, prediction was done by sequential inference through BPNN and Elman models using the collected driving data from a driving simulator and NGSIM. The BP neural network model did a better job predicting lane-changing trajectories under different path sections and generated reliable simulation results. Note that among the various data, the vehicle trajectory is influenced by time headway and the prior position more obviously.

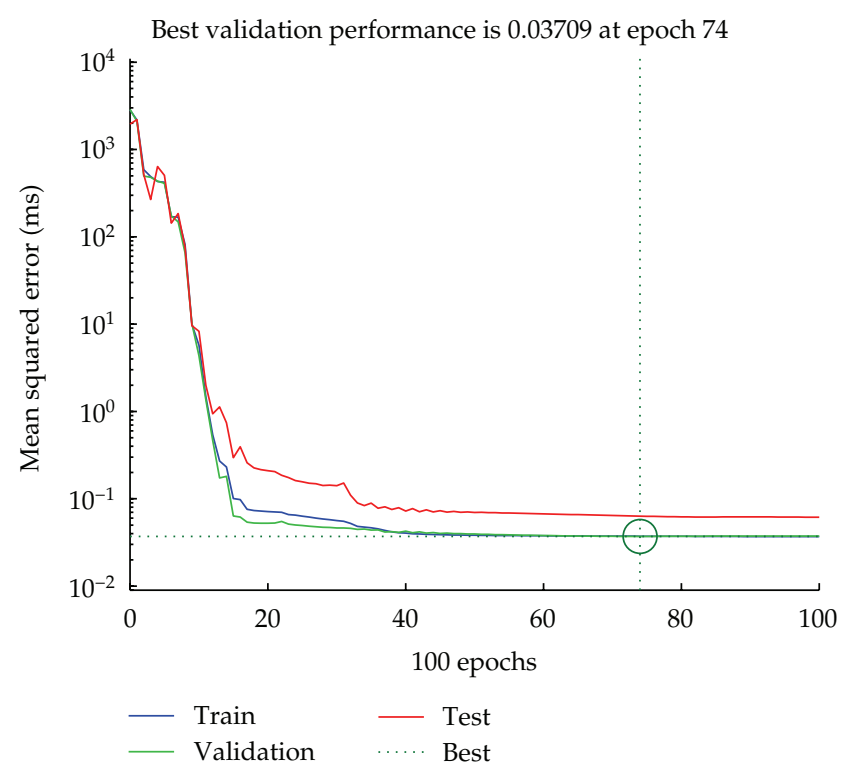

FIGURE 14: Performance curve of BPNN.

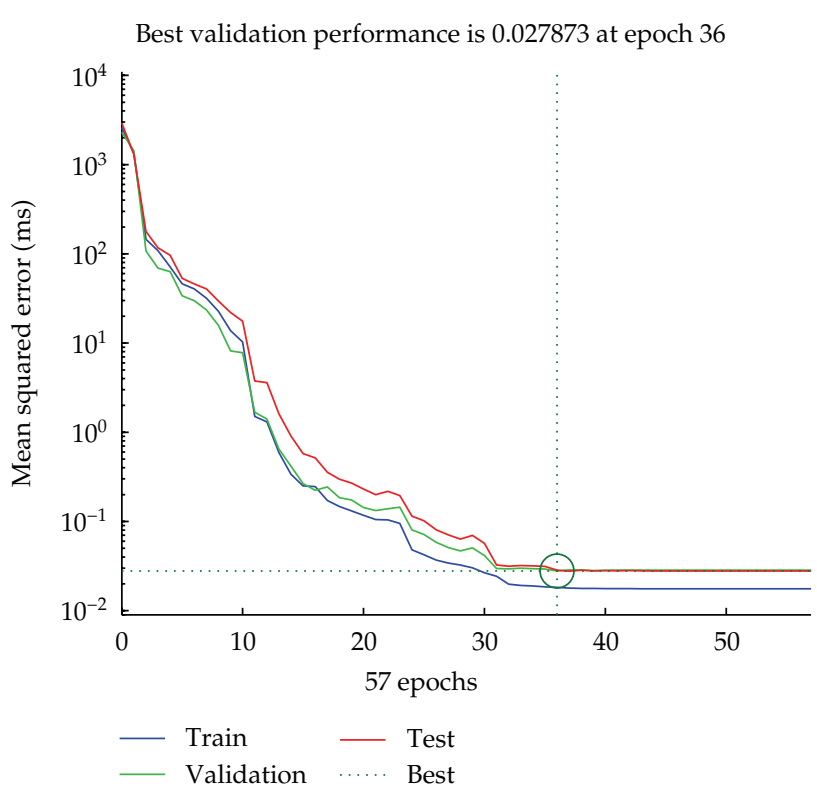

FIGURE 15: Performance curve of Elman Network.

We can use this network model as a basic model or an initial point to create more complex lane-changing models. For future research, perhaps the results of the simulation could be improved by using more inputs, such as the current states of the vehicles and the driver's reaction time. Reaction time could be increased when the driver is under stress or distracted. Therefore, future research should also consider how to incorporate mental workload in the driver behavior model and how to increase the length of the prediction window. One possible method to incorporate the mental workload is to measure the mental workload and define it as a part of the input to the neural network model. Meanwhile, for the future research, we attempt to develop a real-time, 


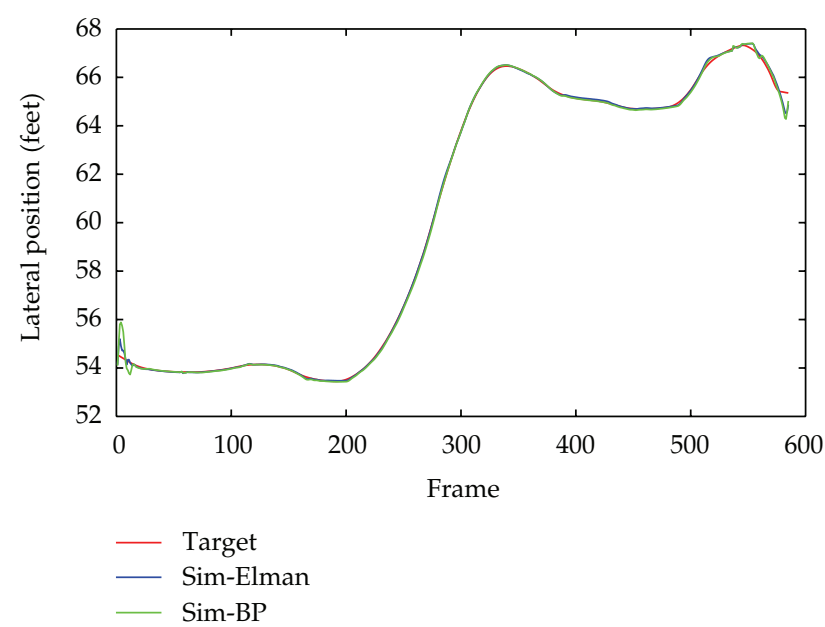

FIGURE 16: Predicted lateral trajectory of the vehicle based on BPNN and Elman Network with 1 s prediction time.

on-road lane-change detector that can anticipate the future driving state. This detector extracts signals from vehicle sensors and preprocesses them into feature vectors, which are then used for offline training and online inference. Finally, the simulation results could be used to assess the safety of lane-changing maneuvers and lay a necessary foundation for further development of the autonomous lane-changing and overtaking assistance systems.

\section{Acknowledgments}

This research was supported in part by National Nature Science Foundation of China under Grant 51010305084, 51110305060, 51210305046, and the Program of Introducing Talents of Discipline to Universities under Grant B12022. The authors also gratefully acknowledge the helpful comments and suggestions of the colleagues from Transportation Research Center of Beijing University of Technology, which have improved the paper.

\section{References}

[1] W. H. Wang, Q. Cao, K. Ikeuchi, and H. Bubb, "Reliability and safety analysis methodology for identification of drivers' erroneous actions," International Journal of Automotive Technology, vol. 11, no. 6, pp. 873-881, 2010.

[2] P. Varaiya, "Smart cars on smart roads: problems of control," IEEE Transactions on Automatic Control, vol. 38, no. 2, pp. 195207, 1993.

[3] M. Peden, R. Scurfield, D. Sleet et al., World Report on Road Traffic Injury Prevention, World Health Organization, Geneva, Switzerland, 2004.

[4] S. E. Lee, E. C. B. Olsen, and W. W. Wienville, A Comprehensive Examination of Naturalistic Lane-Changes, Virginia Tech Transportation Institute I National Highway Transportation Safety Administration, Blacksburg, Va, USA, 2004.

[5] T. Kumagai, Y. Sakaguchi, M. Okuwa et al., "Prediction of driving behavior through probabilistic inference," in Proceedings of the International conference on Engineering Application of Neural Networks (EANN '03), pp. 117-123, Malaga, Spain.
[6] S. Tezuka, H. Soma, and K. Tanifuji, "A study of driver behavior inference model at time of lane change using Bayesian Networks," in Proceedings of the IEEE International Conference on Industrial Technology (ICIT '06), pp. 2308-2313, Mumbai, India, December 2006.

[7] N. Kuge, T. Yamamura, and O. Shimoyama, A Driver Behavior Recognition Method Based on a Driver Model framework, SAE, Warrendale, Pa, USA, 1998.

[8] A. Sathyanarayana, P. Boyraz, and J. H. L. Hansen, "Driver behavior analysis and route recognition by hidden Markov models," in Proceedings of the IEEE International Conference on Vehicular Electronics and Safety (ICVES '08), pp. 276-281, Columbus, Ohio, USA, September 2008.

[9] A. Pentland and L. Andrew, "Modeling and prediction of human behavior," Neural Computation, vol. 11, no. 1, pp. 229242, 1999.

[10] C. C. Macadam and G. E. Johnson, "Application of elementary neural networks and preview sensors for representing driver steering control behaviour," Vehicle System Dynamics, vol. 25, no. 1, pp. 3-30, 1996.

[11] R. M. H. Cheng, J. W. Xiao, and S. LeQuoc, "Neuromorphic controller for AGV steering," in Proceedings of the IEEE International Conference on Robotics and Automation, pp. 2057-2062, May 1992.

[12] R. S. Tomar, S. Verma, and G. S. Tomar, "Prediction of lane change trajectories through neural network," in Proceedings of the International Conference on Computational Intelligence and Communication Networks (CICN '10), pp. 249-253, Bhopal, India, November 2010.

[13] W. Wang, H. Guo, H. Bubb, and K. Ikeuchi, "Numerical simulation and analysis procedure for model-based digital driving dependability in intelligent transport system," KSCE Journal of Civil Engineering, vol. 15, no. 5, pp. 891-898, 2011.

[14] W. Wang, X. Jiang, S. Xia, and Q. Cao, "Incident tree model and incident tree analysis method for quantified risk assessment: an in-depth accident study in traffic operation," Safety Science, vol. 48, no. 10, pp. 1248-1262, 2010.

[15] W. Wang, W. Zhang, H. Guo, H. Bubb, and K. Ikeuchi, "A safetybased approaching behavioural model with various driving characteristics," Transportation Research Part C, vol. 19, no. 6, pp. 1202-1214, 2011.

[16] W. H. Wang, C. X. Ding, G. D. Feng, and X. B. Jiang, "Simulation modelling of longitudinal safety spacing in inter-vehicles dynamics interactions," Journal of Beijing Institute of Technology, vol. 19, supplement 2, pp. 55-60, 2010.

[17] A. H. H. A. Memar, P. Z. H. Bagher, and M. Keshmiri, "Mechanical design and motion planning of a modular reconfigurable robot," in Proceedings of 11th International of Conference on Climbing and Walking Robots and the Support Technologies for Mo, pp. 1090-1097, 2008.

[18] T. Kohonen, "An introduction to neural computing," Neural Networks, vol. 1, no. 1, pp. 3-16, 1988.

[19] E. G. Tsionas, P. G. Michaelides, and A. T. Vouldis, "Global approximations to cost and production functions using artificial neural networks," International Journal of Computational Intelligence Systems, vol. 2, no. 2, pp. 132-139, 2009.

[20] E. Zio, "Neural networks simulation of the transport of contaminants in groundwater," International Journal of Computational Intelligence Systems, vol. 2, no. 3, pp. 267-276, 2009. 
[21] Gowrishankar and P. S. Satyanarayana, "Neural network based traffic prediction for wireless data networks," International Journal of Computational Intelligence Systems, vol. 1, no. 4, pp. 379389, 2008.

[22] B. Yegnanarayana, Artificial Neural Networks, PHI, New Delhi, India, 2005.

[23] T. Kaya, E. Aktaş, I. Topçu, and B. Ülengin, "Modeling toothpaste brand choice: an empirical comparison of artificial neural networks and multinomial probit model," International Journal of Computational Intelligence Systems, vol. 3, no. 5, pp. 674-687, 2010.

[24] M. R. K. Baumann, D. Rosier, and J. F. Krems, "Situation awareness and secondary task performance while driving," in Proceedings of the 7th International Conference on Engineering Psychology and Cognitive Ergonomics (EPCE '07), vol. 4562 of Lecture Notes in Computer Science, pp. 256-263, Beijing, China, 2007. 


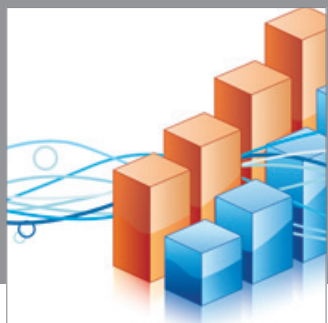

Advances in

Operations Research

mansans

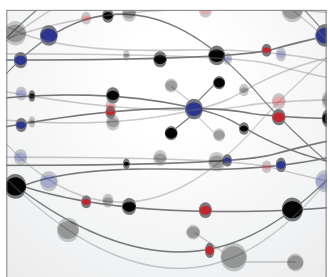

The Scientific World Journal
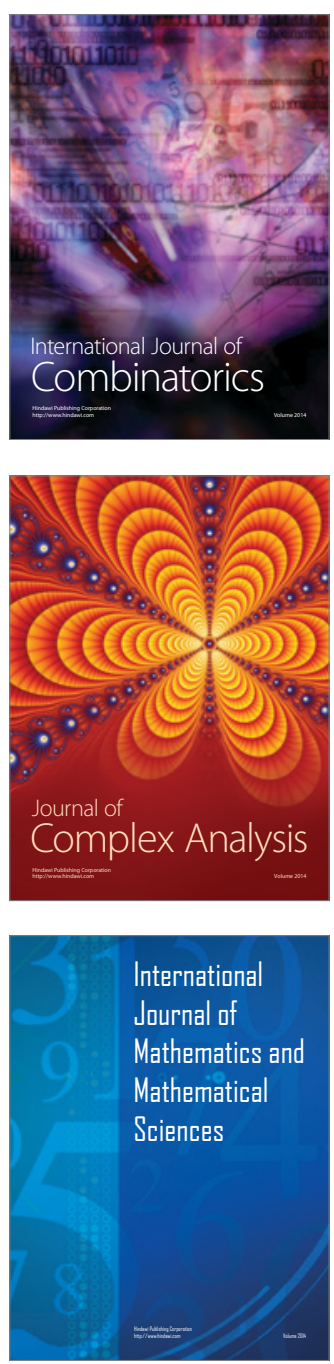
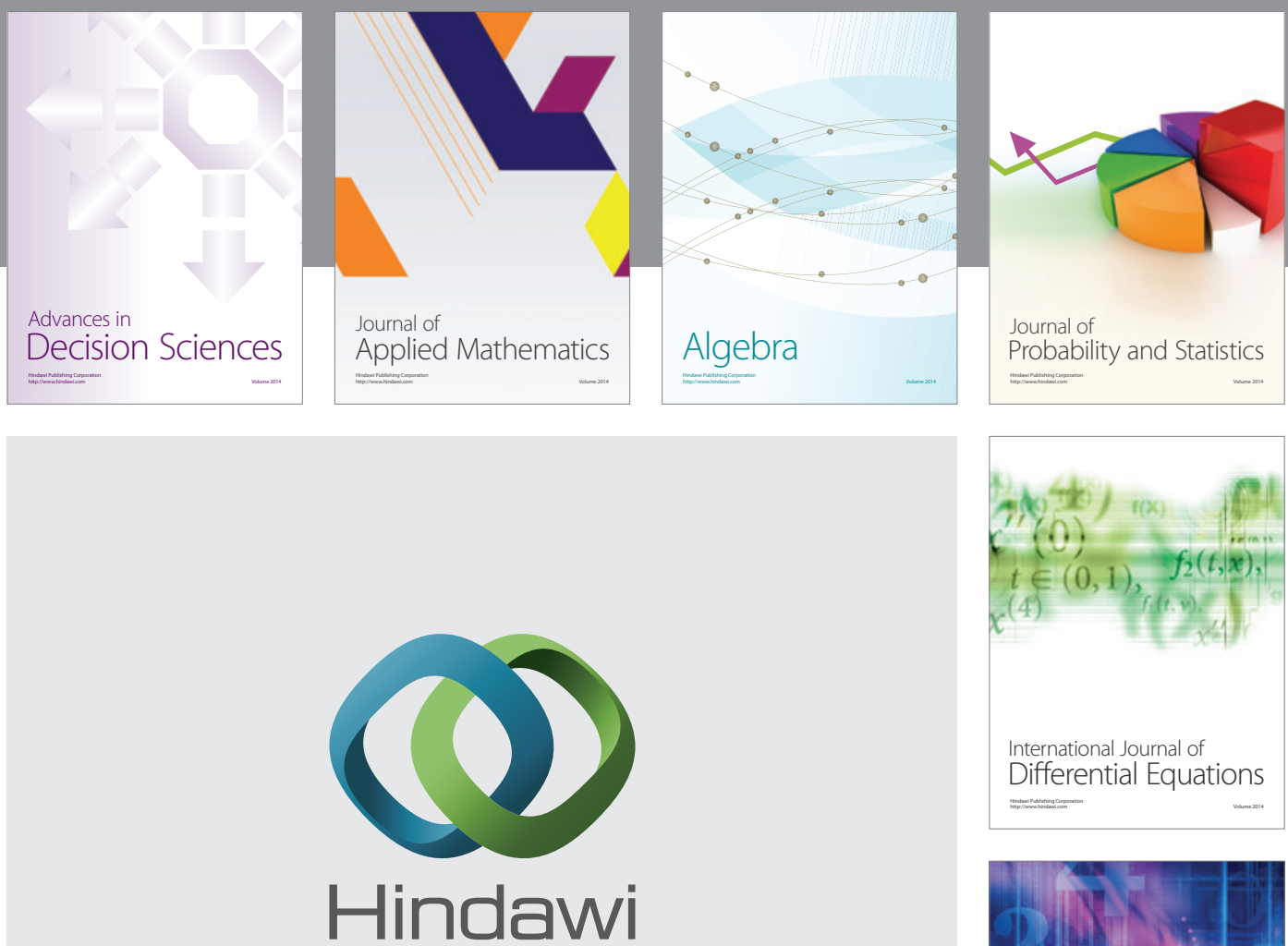

Submit your manuscripts at http://www.hindawi.com
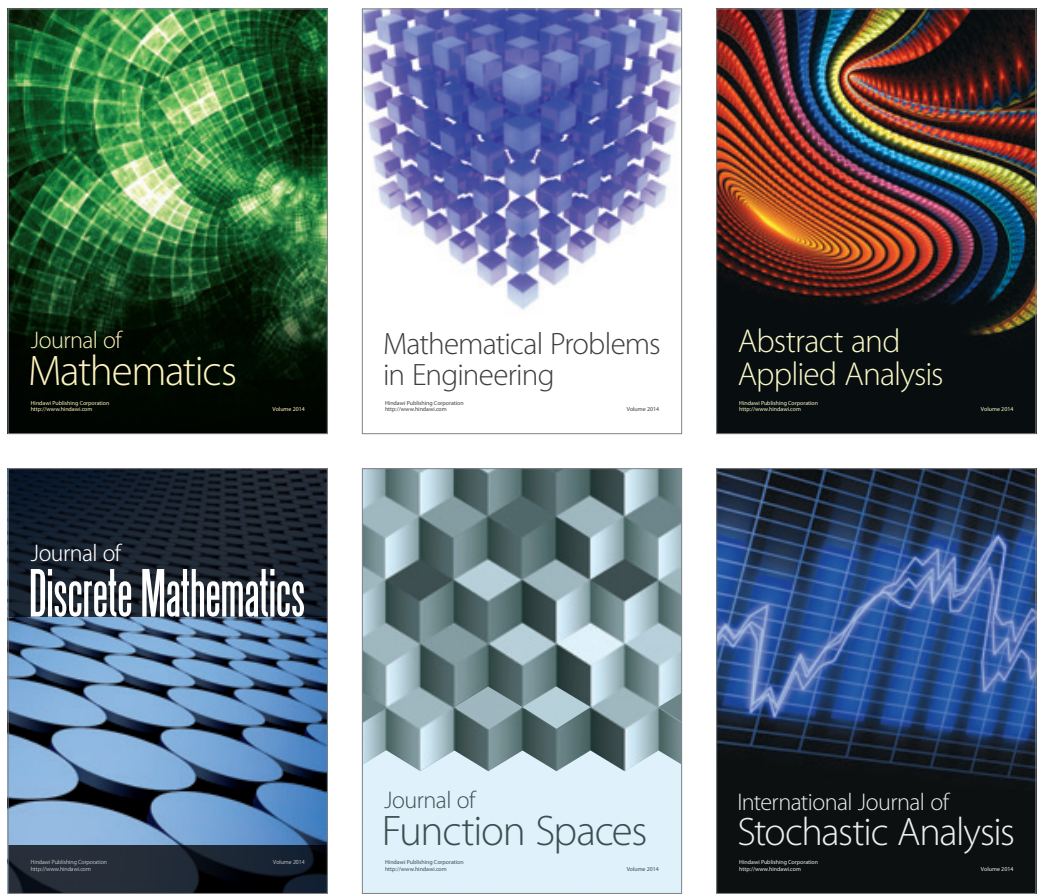

Journal of

Function Spaces

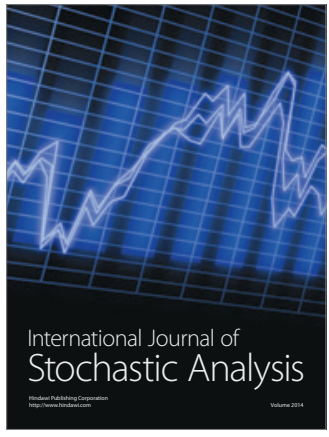

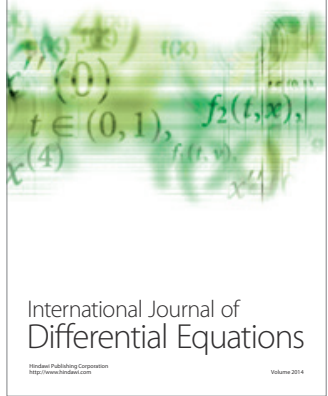
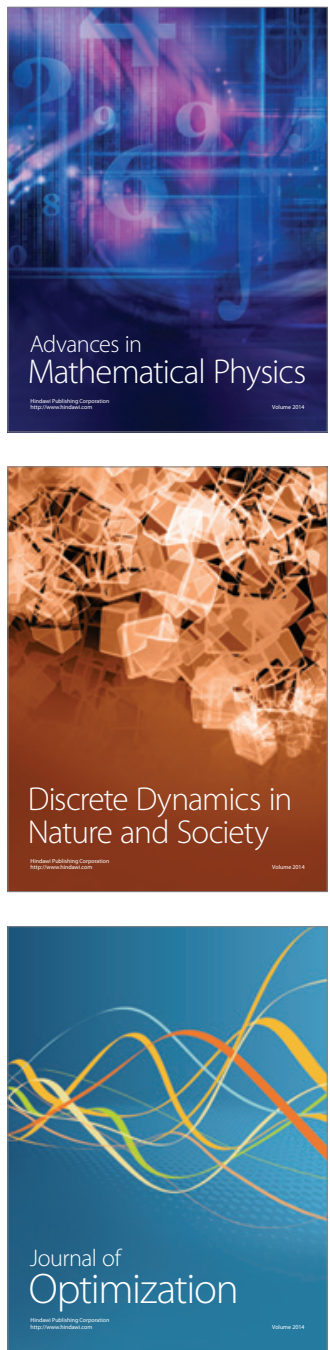